\title{
Brain MR Image Segmentation Using Local and Global Intensity Fitting Active Contours/Surfaces
}

\author{
Li Wang ${ }^{1}$, Chunming $\mathrm{Li}^{2, \star}$, Quansen $\mathrm{Sun}^{1}$, Deshen $\mathrm{Xia}^{1}$, and Chiu-Yen $\mathrm{Kao}^{3}$ \\ ${ }^{1}$ School of Computer Science \& Technology, \\ Nanjing University of Science and Technology, China \\ ${ }^{2}$ Institute of Imaging Science, Vanderbilt University, USA \\ chunming. li@vanderbilt.edu \\ ${ }^{3}$ Department of Mathematics, The Ohio State University, USA
}

\begin{abstract}
In this paper, we present an improved region-based active contour/surface model for $2 \mathrm{D} / 3 \mathrm{D}$ brain MR image segmentation. Our model combines the advantages of both local and global intensity information, which enable the model to cope with intensity inhomogeneity. We define an energy functional with a local intensity fitting term and an auxiliary global intensity fitting term. In the associated curve evolution, the motion of the contour is driven by a local intensity fitting force and a global intensity fitting force, induced by the local and global terms in the proposed energy functional, respectively. The influence of these two forces on the curve evolution is complementary. When the contour is close to object boundaries, the local intensity fitting force became dominant, which attracts the contour toward object boundaries and finally stops the contour there. The global intensity fitting force is dominant when the contour is far away from object boundaries, and it allows more flexible initialization of contours by using global image information. The proposed model has been applied to both 2D and 3D brain MR image segmentation with promising results.
\end{abstract}

\section{Introduction}

The segmentation of the brain magnetic resonance (MR) images into white matter (WM), gray matter (GM), and cerebral spinal fluid (CSF) has been an important and fundamental step in research and clinical applications, including diagnosis of pathology, presurgical planning and computer integrated surgery. A major difficulty in segmentation of MR images is the intensity inhomogeneities due to the radio-frequency coils or acquisition sequences.

Active contour models have been widely used in medical image segmentation [1234567891011. The existing active contour models can be categorized into two classes: edge-based models [1,3/4] and region-based models [5]6/7891011]. Edge-based models may suffer from boundary leakage problem

\footnotetext{
* Corresponding author.
}

D. Metaxas et al. (Eds.): MICCAI 2008, Part I, LNCS 5241, pp. 384 392, 2008.

(C) Springer-Verlag Berlin Heidelberg 2008 
for brain MR images, in which some parts of WM and GM boundaries are quite fuzzy due to low contrast there. Region-based models have better performance than edge-based models in the presence of weak boundaries. However, most of region-based models [5/6/78] tend to rely on intensity homogeneity. For example, the well-known piecewise constant (PC) models [7108] are based on the assumption that image intensities are statistically homogeneous (roughly a constant) in each region, therefore they fail to segment MR images with intensity inhomogeneity. In [10] and [11], two similar active contour models were proposed to minimize the Mumford-Shah functional [12. These models, widely known as piecewise smooth (PS) models, have exhibited certain capability of handling intensity inhomogeneity. However, the computational cost of the PS model is rather expensive due to the complicated procedures 13. Moreover, to the best of our knowledge, the PS models have not been used to segment brain MR images.

Recently, Li et al. proposed a local binary fitting (LBF) model 14 15 to overcome intensity inhomogeneity. The LBF model draws upon local intensity information, which enables the model to cope with intensity inhomogeneity. With accurate local intensity information, the LBF model is able to recover object boundaries precisely. Some related methods were recently proposed in [1316, which have similar capability of handling intensity inhomogeneity as the LBF model. However, these methods [14151316] are to some extent sensitive to initialization, which limit their practical applications.

In this paper, we propose an improved active contour/surface model, which combines the advantages of both local and global intensity information. We define an energy functional with two terms: one is a local intensity fitting term and the other is an auxiliary global intensity fitting term. The local intensity fitting term induces a local force to attract the contours and stop it at object boundaries, which enables the model to cope with intensity inhomogeneity. The global intensity fitting term drives the motion of the contour far away from object boundaries, and therefore allows for flexible initialization of the contours.

\section{Background}

\subsection{Chan-Vese Model}

Chan and Vese [7] proposed an active contour approach to the Mumford-Shah problem [12 for a special case where the original image is a piecewise constant function. The global data fitting term in the Chan-Vese (CV) model is defined as follows

$$
\mathcal{F}^{\mathrm{CV}}\left(c_{1}, c_{2}, C\right)=\int_{\text {outside }(C)}\left(I-c_{1}\right)^{2} d \mathbf{x} d \mathbf{y}+\int_{\text {inside }(C)}\left(I-c_{2}\right)^{2} d \mathbf{x} d \mathbf{y}
$$

where outside $(C)$ and inside $(C)$ represent the regions outside and inside the contour $C$, respectively, and $c_{1}$ and $c_{2}$ are two constants that fit the image intensities in the entire regions outside $(C)$ and inside $(C)$. Obviously, the constants $c_{1}$ and 
$c_{2}$ that best fit the intensity are not accurate, if the intensities in each region separated by $C$ are inhomogeneous. Without taking local image information into account, the CV model generally fails to segment images with intensity inhomogeneity. Likewise, more general piecewise constant models in a multiphase level set framework [108] are not applicable for such images either.

\subsection{Local Binary Fitting Model}

To overcome the difficulty caused by intensity inhomogeneities, Li et al. proposed the local binary fitting (LBF) model, which utilizes the local intensity information [1415. Two spatially varying fitting functions $f_{1}(\mathbf{x})$ and $f_{2}(\mathbf{x})$ are introduced to approximate the local intensities on the two sides of the contour. In the LBF model, the local data fitting term was defined as follows

$$
\begin{aligned}
\mathcal{F}^{\mathrm{LBF}}\left(\phi, f_{1}, f_{2}\right) & =\lambda_{1} \int\left[\int K_{\sigma}(\mathbf{x}-\mathbf{y})\left|I(\mathbf{y})-f_{1}(\mathbf{x})\right|^{2} H(\phi(\mathbf{y})) d \mathbf{y}\right] d \mathbf{x} \\
& +\lambda_{2} \int\left[\int K_{\sigma}(\mathbf{x}-\mathbf{y})\left|I(\mathbf{y})-f_{2}(\mathbf{x})\right|^{2}(1-H(\phi(\mathbf{y}))) d \mathbf{y}\right] d \mathbf{x}
\end{aligned}
$$

where $H$ is Heaviside function, and $K_{\sigma}$ is a Gaussian kernel with standard deviation $\sigma$. Due to the localization property of the kernel function, the local data fitting energy is dominated by the intensities $I(\mathbf{y})$ in a neighborhood of $\mathbf{x}$. This localization property enables the LBF model to deal with intensity inhomogeneity. Moreover, the LBF model can recover object boundaries more precisely than other region-based models without using local intensity information. However, at the cost of introducing the localization property, the LBF model becomes more sensitive to initialization than the PC models.

\section{Local and Global Intensity Fitting Energy}

Our method combines the advantages of the CV model and the LBF model by taking the local and global intensity information into account. In this section, we will detail our active contour model based on local and global intensity fitting (LGIF) to handle intensity inhomogeneity.

The local intensity fitting energy [14] is defined as follows, which is similar with Eq. (2):

$$
\begin{aligned}
\mathcal{E}^{\mathrm{LIF}}\left(\phi, f_{1}, f_{2}\right) & =\int\left[\int K_{\sigma}(\mathbf{x}-\mathbf{y})\left|I(\mathbf{y})-f_{1}(\mathbf{x})\right|^{2} H(\phi(\mathbf{y})) d \mathbf{y}\right] d \mathbf{x} \\
& +\int\left[\int K_{\sigma}(\mathbf{x}-\mathbf{y})\left|I(\mathbf{y})-f_{2}(\mathbf{x})\right|^{2}(1-H(\phi(\mathbf{y}))) d \mathbf{y}\right] d \mathbf{x}
\end{aligned}
$$

We use the CV model's global intensity fitting energy

$$
\mathcal{E}^{\mathrm{GIF}}\left(\phi, c_{1}, c_{2}\right)=\int\left|I(\mathbf{x})-c_{1}\right|^{2} H(\phi(\mathbf{x})) d \mathbf{x}+\int\left|I(\mathbf{x})-c_{2}\right|^{2}(1-H(\phi(\mathbf{x}))) d \mathbf{x}
$$


Now, we define the following energy functional

$$
\mathcal{E}^{\mathrm{LGIF}}\left(\phi, c_{1}, c_{2}, f_{1}, f_{2}\right)=(1-\omega) \mathcal{E}^{\mathrm{LIF}}\left(\phi, f_{1}, f_{2}\right)+\omega \mathcal{E}^{\mathrm{GIF}}\left(\phi, c_{1}, c_{2}\right)
$$

where $\omega$ is a positive constant $(0 \leq \omega \leq 1)$. When the intensity inhomogeneity in the image is severe, the parameter value $\omega$ should be chosen small enough. For more accurate computation involving the level set function, we need to regularize the level set function by penalizing its deviation from a signed distance function [17, which can be characterized by the following energy functional

$$
\mathcal{P}(\phi)=\int \frac{1}{2}(|\nabla \phi(\mathbf{x})|-1)^{2} d \mathbf{x}
$$

In addition, it is necessary to smooth the zero level set contour by penalizing its length, which is computed by

$$
\mathcal{L}(\phi)=\int|\nabla H(\phi(\mathbf{x}))| d \mathbf{x}
$$

Now, we define the entire energy functional

$$
\mathcal{F}\left(\phi, f_{1}, f_{2}, c_{1}, c_{2}\right)=\mathcal{E}^{\mathrm{LGIF}}\left(\phi, c_{1}, c_{2}, f_{1}, f_{2}\right)+\nu \mathcal{L}(\phi)+\mu \mathcal{P}(\phi)
$$

It can be shown that the optimal fitting functions $f_{1}$ and $f_{2}$ and constants $c_{1}$ and $c_{2}$ that minimize the energy (8) are given by

$$
\begin{gathered}
f_{1}(\mathbf{x})=\frac{K_{\sigma}(\mathbf{x}) *[H(\phi(\mathbf{x})) I(\mathbf{x})]}{K_{\sigma}(\mathbf{x}) * H(\phi(\mathbf{x}))}, \quad f_{2}(\mathbf{x})=\frac{K_{\sigma}(\mathbf{x}) *[(1-H(\phi(\mathbf{x}))) I(\mathbf{x})]}{K_{\sigma}(\mathbf{x}) *[1-H(\phi(\mathbf{x}))]} \\
c_{1}=\frac{\int I(\mathbf{x}) H(\phi(\mathbf{x})) d \mathbf{x}}{\int H(\phi(\mathbf{x})) d \mathbf{x}}, \quad c_{2}=\frac{\int I(\mathbf{x})(1-H(\phi(\mathbf{x}))) d \mathbf{x}}{\int(1-H(\phi(\mathbf{x}))) d \mathbf{x}}
\end{gathered}
$$

Minimization of the energy functional $\mathcal{F}$ in Eq. (8) with respect to $\phi$ is achieved by solving the gradient descent flow equation

$$
\frac{\partial \phi}{\partial t}=-\delta(\phi)\left(F_{1}+F_{2}\right)+\nu \delta(\phi) \operatorname{div}\left(\frac{\nabla \phi}{|\nabla \phi|}\right)+\mu\left(\nabla^{2} \phi-\operatorname{div}\left(\frac{\nabla \phi}{|\nabla \phi|}\right)\right)
$$

where $\delta(\cdot)$ is the Dirac delta function, and $F_{1}=(1-\omega)\left[\int K_{\sigma}(\mathbf{y}-\mathbf{x}) \mid I(\mathbf{x})-\right.$ $\left.\left.f_{1}(\mathbf{y})\right|^{2} d \mathbf{y}-\int K_{\sigma}(\mathbf{y}-\mathbf{x})\left|I(\mathbf{x})-f_{2}(\mathbf{y})\right|^{2} d \mathbf{y}\right], F_{2}=\omega\left[\left(I-c_{1}\right)^{2}-\left(I-c_{2}\right)^{2}\right]$. We call $F_{1}$ and $F_{2}$ the local intensity fitting (LIF) force and global intensity fitting (GIF) force, respectively.

\subsection{Influence of the LIF and GIF Forces}

The influence of the LIF force and GIF force on the curve evolution is complementary. When the contour is near object boundaries, the LIF force is dominant, which attracts the contour toward object boundaries and finally stops the contour there. Therefore, the location of the final contour is determined by the LIF 


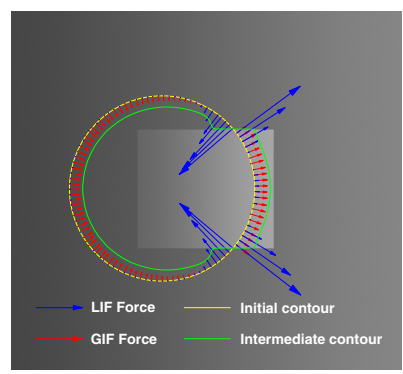

Fig. 1. Influence of LIF and GIF forces

force. When the contour is far away from object boundaries, the GIF force is dominant, while the LIF force is close to zero. This can be illustrated by an example shown in Fig. 1. The initial contour and the contour after 10 iterations are plotted in yellow and green respectively. The blue and red arrows represent the LIF and GIF force, respectively. The magnitude of the force is represented by the length of the arrow. It can be seen that the LIF force is dominant near the object boundaries, while the GIF force is dominant at locations far away from object boundaries.

\subsection{Extension to Multi-phase Formulation}

In this subsection, we extend the LGIF model to a multi-phase level set formulation, so that WM, GM, and CSF can be segmented simultaneously. By using $n$ level set functions, at most $2^{n}$ regions can be segmented [10]. In a four-phase level set representation, two level set functions $\phi_{1}, \phi_{2}$ are used to define the following four regions: $\left\{\phi_{1}>0, \phi_{2}>0\right\},\left\{\phi_{1}>0, \phi_{2}<0\right\},\left\{\phi_{1}<0, \phi_{2}>0\right\}$, $\left\{\phi_{1}<0, \phi_{2}<0\right\}$. We define the following energy functional

$$
\begin{aligned}
\mathcal{F} & =(1-\omega) \sum_{i=1}^{4} \iint K_{\sigma}(\mathbf{x}-\mathbf{y})\left|I(\mathbf{y})-f_{i}(\mathbf{x})\right|^{2} m_{i}(\mathbf{y}) d \mathbf{y} d \mathbf{x} \\
& +\omega \sum_{i=1}^{4} \int\left|I(\mathbf{x})-c_{i}\right|^{2} m_{i}(\mathbf{x}) d \mathbf{x} \\
& +\nu\left(\mathcal{L}\left(\phi_{1}\right)+\mathcal{L}\left(\phi_{2}\right)\right)+\mu\left(\mathcal{P}\left(\phi_{1}\right)+\mathcal{P}\left(\phi_{2}\right)\right)
\end{aligned}
$$

where $m_{1}=H\left(\phi_{1}\right) H\left(\phi_{2}\right), m_{2}=H\left(\phi_{1}\right)\left(1-H\left(\phi_{2}\right)\right), m_{3}=\left(1-H\left(\phi_{1}\right)\right) H\left(\phi_{2}\right)$, $m_{4}=\left(1-H\left(\phi_{1}\right)\right)\left(1-H\left(\phi_{2}\right)\right)$.

It can be shown that the optimal fitting functions $f_{1}, \cdots, f_{4}$ and constants $c_{1}, \cdots, c_{4}$ that minimize the energy (12) are given by

$$
f_{i}(\mathbf{x})=\frac{K_{\sigma}(\mathbf{x}) *\left[m_{i} I(\mathbf{x})\right]}{K_{\sigma}(\mathbf{x}) * m_{i}}, \quad c_{i}=\frac{\int_{\Omega} I(\mathbf{x}) m_{i} d \mathbf{x}}{\int_{\Omega} m_{i} d \mathbf{x}}, \quad i=1, \cdots, 4
$$


Minimization of the energy functional $\mathcal{F}$ in Eq. (12) with respect to $\phi_{1}$ and $\phi_{2}$ is achieved by solving the gradient descent flow equations

$$
\begin{aligned}
\frac{\partial \phi_{1}}{\partial t} & =-\delta\left(\phi_{1}\right)\left(H\left(\phi_{2}\right)\left(e_{1}-e_{3}\right)+\left(1-H\left(\phi_{2}\right)\right)\left(e_{2}-e_{4}\right)\right)+\nu \delta\left(\phi_{1}\right) \operatorname{div}\left(\frac{\nabla \phi_{1}}{\left|\nabla \phi_{1}\right|}\right) \\
& +\mu\left(\nabla^{2} \phi_{1}-\operatorname{div}\left(\frac{\nabla \phi_{1}}{\left|\nabla \phi_{1}\right|}\right)\right) \\
\frac{\partial \phi_{2}}{\partial t} & =-\delta\left(\phi_{2}\right)\left(H\left(\phi_{1}\right)\left(e_{1}-e_{2}\right)+\left(1-H\left(\phi_{1}\right)\right)\left(e_{3}-e_{4}\right)\right)+\nu \delta\left(\phi_{2}\right) \operatorname{div}\left(\frac{\nabla \phi_{2}}{\left|\nabla \phi_{2}\right|}\right) \\
& +\mu\left(\nabla^{2} \phi_{2}-\operatorname{div}\left(\frac{\nabla \phi_{2}}{\left|\nabla \phi_{2}\right|}\right)\right)
\end{aligned}
$$

where $e_{i}(\mathbf{x})=(1-\omega) \int K_{\sigma}(\mathbf{y}-\mathbf{x})\left|I(\mathbf{x})-f_{i}(\mathbf{y})\right|^{2} d \mathbf{y}+\omega\left|I(\mathbf{x})-c_{i}\right|^{2}$.

\section{Experimental Results}

In this paper, we use the following default setting of the parameters $\sigma=3.0$, $\mu=1.0, \nu=0.001 \times 255 \times 255$, time step $\Delta t=0.1$, and $\omega=0.01$. When the intensity inhomogeneity in the image is more severe, the parameter value $\omega$ should be chosen smaller. We first apply our multi-phase model to segment a MR image from McGill Brain Web [18] with noise level 3\%, and intensity nonuniformity (INU) 40\%, as shown in the first row of Fig. 2, We have increased INU to test the validity of our method to handle intensity inhomogeneity. The second row shows a real brain MR image with obvious intensity inhomogeneity. For this image, we set $\omega=0.005$. In fact, some intensities of the white matter in the upper part are even lower than those of the gray matter in the lower part. Nevertheless, our method achieves satisfactory segmentation results for these two images, as shown in the second column of Fig. 2. To demonstrate the advantage of our model in terms of accuracy, we compare it with the PC model [10]. We used Wells et al.'s algorithm [19] to correct intensity inhomogeneity before we applied the PC models. The third column shows the results of the PC model. We use the Jaccard similarity 20] as a metric to evaluate the performance of image segmentation algorithms. For this McGill Brain Web image, the JS for WM, GM and CSF obtained by the PC model are $0.87,0.79$, and 0.77 respectively. The corresponding JS coefficients of the segmentation result obtained by our method are $0.91,0.81$, and 0.82 respectively. Compared with the results obtained by the PC model, the results of our method are more accurate.

Fig. 3 shows the surfaces of the GM and WM segmentation of real 3D brain MR images with obvious intensity inhomogeneity. The upper and lower row show the results obtained by PC model and our model, respectively. It can be seen that GM obtained by the PC model is a little bit thinner at the top of the 

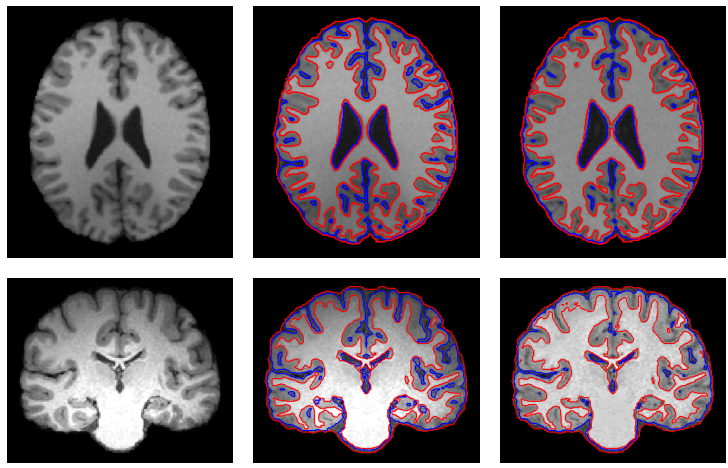

Fig. 2. Results for brain MR images. The red and blue curves are zero level set of $\phi_{1}$ and $\phi_{2}$. Column 1: Original images; Column 2: Results of our model; Column 3: Results of PC model applying to corrected images.
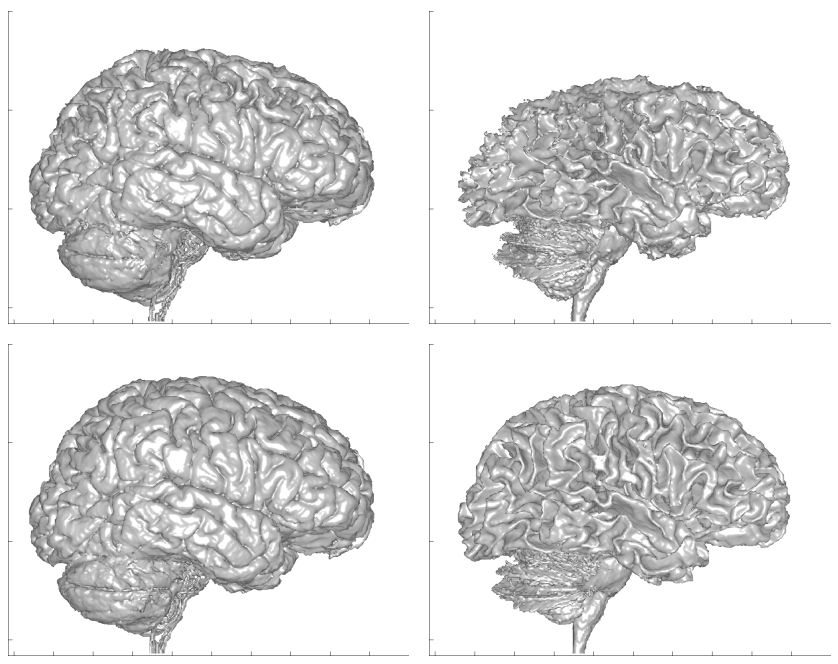

Fig. 3. Results for 3D brain MR images. Row 1: GM and WM surfaces obtained by the PC model; Row 2: GM and WM surfaces obtained by our model.

brain and WM is seriously misclassified as GM. While the surfaces obtained by our method are more accurate. To demonstrate the advantage of our method clearly, we show two sagittal slices and the corresponding contours obtained by the PC model and our method in Fig. 4. It can be clearly seen that the PC model does not correctly segment images: part of the WM is incorrectly identified as the GM, while part of the GM is labeled as the WM. By contrast, our method recovers the boundaries of WM, GM, and CSF accurately. 

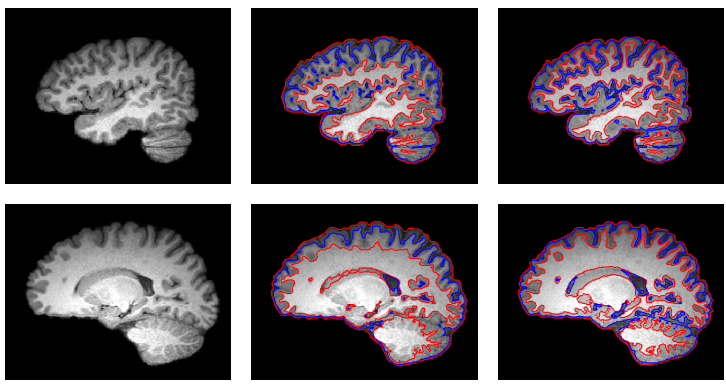

Fig. 4. Sagittal view of 3D segmentation of Fig. 3. Column 1: Original images; Column 2: Results of the PC model; Column 3: Results of our method.

\section{Conclusion}

In this paper, we provide an improved region-based active contour/surface model for $2 \mathrm{D}$ and $3 \mathrm{D}$ brain $\mathrm{MR}$ image segmentation by drawing upon both local and global intensity information, which is better adapted to the intensity inhomogeneity problem. Experimental results demonstrate desirable performance of our method for brain MR images with intensity inhomogeneity.

\section{References}

1. Kass, M., Witkin, A., Terzopoulos, D.: Snakes: active contour models. Int'l J. Comp. Vis. 1, 321-331 (1987)

2. Caselles, V., Catte, F., Coll, T., Dibos, F.: A geometric model for active contours in image processing. Numer. Math. 66, 1-31 (1993)

3. Kimmel, R., Amir, A., Bruckstein, A.: Finding shortest paths on surfaces using level set propagation. IEEE Trans. Patt. Anal. Mach. Intell. 17, 635-640 (1995)

4. Xu, C., Prince, J.: Snakes, shapes, and gradient vector flow. IEEE Trans. Imag. Proc. 7(3), 359-369 (1998)

5. Ronfard, R.: Region-based strategies for active contour models. Int'l. J. Comp. Vis. 13, 229-251 (1994)

6. Samson, C., Blanc-Feraud, L., Aubert, G., Zerubia, J.: A variational model for image classification and restoration. IEEE Trans. Patt. Anal. Mach. Intell. 22(5), 460-472 (2000)

7. Chan, T., Vese, L.: Active contours without edges. IEEE Trans. Imag. Proc. 10, 266-277 (2001)

8. Paragios, N., Deriche, R.: Geodesic active regions and level set methods for supervised texture segmentation. Int'l. J. Comp. Vis. 46, 223-247 (2002)

9. Rousson, M., Cremers, D.: Implicit active shape models for 3D segmentation in MR imaging. In: Barillot, C., Haynor, D.R., Hellier, P. (eds.) MICCAI 2004. LNCS, vol. 3216, pp. 209-216. Springer, Heidelberg (2004)

10. Vese, L., Chan, T.: A multiphase level set framework for image segmentation using the Mumford and Shah model. Int'l. J. Comp. Vis. 50, 271-293 (2002) 
11. Tsai, A., Yezzi, A., Willsky, A.S.: Curve evolution implementation of the MumfordShah functional for image segmentation, denoising, interpolation, and magnification. IEEE Trans. Imag. Proc. 10, 1169-1186 (2001)

12. Mumford, D., Shah, J.: Optimal approximations by piecewise smooth functions and associated variational problems. Commun. Pure Appl. Math. 42, 577-685 (1989)

13. Piovano, J., Rousson, M., Papadopoulo, T.: Efficient segmentation of piecewise smooth images. In: Scale Space and Variational Methods in Computer Vision, pp. 709-720 (2007)

14. Li, C., Kao, C., Gore, J., Ding, Z.: Implicit active contours driven by local binary fitting energy. In: IEEE Conference on Computer Vision and Pattern Recognition(CVPR) (2007)

15. Li, C., Kao, C., Gore, J., Ding, Z.: Minimization of region-scalable fitting energy for image segmentation. IEEE Trans. Imag. Proc. (to appear)

16. Brox, T., Cremers, D.: On the statistical interpretation of the piecewise smooth Mumford-Shah functional. In: Sgallari, F., Murli, A., Paragios, N. (eds.) SSVM 2007. LNCS, vol. 4485, pp. 203-213. Springer, Heidelberg (2007)

17. Li, C., Xu, C., Gui, C., Fox, M.D.: Level set evolution without re-initialization: A new variational formulation. In: IEEE Conference on Computer Vision and Pattern Recognition(CVPR), vol. 1, pp. 430-436 (2005)

18. http://www.bic.mni.mcgill.ca/brainweb/

19. Wells, W., Grimson, E., Kikinis, R., Jolesz, F.: Adaptive segmentation of MRI data. IEEE Trans. Med. Imag. 15(4), 429-442 (1996)

20. Jaccard, P.: The distribution of flora in the alpine zone. New Phytol. 11(2), 37-50 (1912) 